
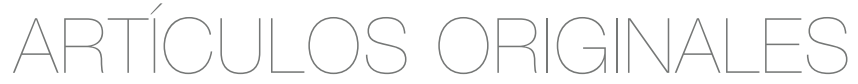

\title{
Editorial.
}

\section{La reflexión de los profesores sobre el currículo y su propia actividad de enseñanza: algunos retos en la educación de hoy}

\section{DIEGO PÉREZ*}

La emergencia de nuevas necesidades en la sociedad del conocimiento ha impuesto el reto a las instituciones de educación superior y a sus profesores de (re)pensar ${ }^{1}$ los procesos de formación de manera constante a nivel teórico, práctico, metodológico, gnoseológico y ontológico.

De igual manera tanto las organizaciones como las universidades se han impuesto como meta que sus estudiantes, próximos profesionales, vivencien los valores propios de la sociedad y que se den las condiciones para una educación que busque el desarrollo (Davídov, 1988; Davídov y Márkova, 1987). Este desarrollo no solo corresponde al carácter 'evolutivo' en las formas de concebir al mundo sino a la expresión de la apropiación de los saberes construidos culturalmente a través de las actividades que realizan los estudiantes en edad escolar (en este caso, en edad de formación universitaria). Este panorama, lejos de ser desalentador, supone un trabajo arduo en torno a las preguntas por el modelo pedagógico y el currículo; considerando a este último como una construcción colectiva que atiende a los intereses sociales y culturales de los sujetos involucrados, es decir, como un organismo vivo (Silva, 2010).
COMO CITAR ESTE ARTÍCULO

How to cite this article:

Pérez, D. (2021). Editorial. La reflexión de los profesores sobre el currículo y su propia actividad de enseñanza: algunos retos en la educación de hoy. Revista Perspectiva Empresarial, 8(1), 3-6.

* Doctor en Educación. Fundación Universitaria CEIPA, Sabaneta, Colombia. E-mail: diego.perez@ceipa.edu.co. ORCID: 0000-0002-4437-9206. Google Scholar: https://scholar.google.com/citations?hl=pt-PT\&user=CPaBQfMAAAAJ.

1. El uso de los prefijos dentro de paréntesis sugiere que el verbo ajustado puede hacerse una y otra vez. Este uso ya ha sido empleado en trabajos como el de Pérez (2020). 


\section{La reflexión constante sobre el currículo: una necesidad}

Una mirada tradicional sobre el currículo se fundamenta en considerar a estecomo un compendio de saberes, normas, derechos, deberes y aspectos de tipo político, social, ético entre otros, en el marco de unos intereses particulares. Esta perspectiva de currículo - obsoleta por demás- ha sido cuestionada en las últimas décadas; reivindicando que el currículo como respuesta a las necesidades de la sociedad debe ser dinámico, debe responder a necesidades e intereses colectivos y debe atender - como organismo vivo-a la dialéctica constante con el medio en el que fue concebido.

El currículo no puede ser pensado únicamente como la "carta de navegación" de una institución sino que debe ser un integrante más del día a día de los estudiantes, los profesores, los directivos y los demás integrantes de la comunidad educativa. Además el currículo no es un documento terminado sino que debe estar abierto a la discusión, la reflexión y la (re)constitución constante.

Las demandas de la sociedad del conocimiento han hecho que las empresas muten y se muevan y sobrevivan a las oleadas de información (verdadera o falsa), las demandas de sus clientes, la competencia y el uso de los datos de sus clientes y colaboradores para sostenerse en el tiempo. Si este es el panorama que ofrece la sociedad, la universidad - como lugar llamado a formar a los profesionales del presente y del futuro- no puede ser indiferente a estos cambios; esto implica, además, una revisión dialéctica de adentro hacia afuera y viceversa (Biggs and Tang, 2011; Scott, 2015; Stößlein and Kanet, 2016).

\section{La reflexión sobre la actividad de enseñanza y el currículo: una actividad docente}

Pensar en los cambios constantes que demanda la sociedad del conocimiento, en aspectos didácticos y metodológicos, implica que tanto directivos como profesores estén llamados a reflexionar e investigar sobre su propia práctica.
La búsqueda de soluciones a esta problemática pone de manifiesto algunas tensiones para los profesores, sobre todo en cuanto al diseño curricular de sus cursos (Morosini, 2000). Estas tensiones se manifiestan en las presiones en cuanto a: ¿qué saberes y competencias se deben evidenciar en un curso?; ¿qué tiempos se deben invertir en una u otra competencia?; ¿qué competencias se deben privilegiar en esta área de conocimiento y cuáles dejar para otras áreas?; causando en los profesores la necesidad de constituir grupos de colegas que se piensen, desde la colectividad, los posibles caminos de formación para sus estudiantes (Zabalza, 2004; Moretti e Moura, 2010; Guzmán y Quimbayo, 2012; Junges e Behrens, 2016).

En este contexto se propone la reflexión constante de los profesores sobre sus propias actividades de enseñanza como una posibilidad para pensar, desde la colectividad, en nuevas apuestas para la enseñanza de los objetos de conocimiento (Imbernón, 1994; Alarcão, 1996; Almeida e Pimenta, 2014; Grillo, 2010). Esta reflexión pasa por cuestionarse, individual y colectivamente, cuál o cuáles aportes se pueden hacer desde el área de conocimiento desde la que se enseña. En este sentido para Grillo (2000),

\begin{abstract}
las condiciones de enseñanza cambian día a día y no existe la seguridad de lo que "es correcto". En esta perspectiva, el profesor necesita ser un investigador que cuestione su pensamiento ysu práctica, actué reflexivamente en el ambiente dinámico, tome decisiones y cree respuestas más adecuadas porque son construidas en una situación concreta propia. (p. 75)
\end{abstract}

Este profesor reflexivo e investigador es un sujeto que - en la medida de sus condiciones y posibilidades - apunta caminos para producir cambios reales y sustanciales en sus planeaciones, en sus materiales metodológicos, en el diseño de sus clases, en el diálogo con sus estudiantes y colegas y en su postura sobre los saberes de las otras áreas. Así, continúa Grillo (2000) exponiendo que:

el profesor, como sujeto reflexivo que es, se inclina sobre el contenido de su propia experiencia, la examina, la relaciona con otras y la analiza a la luz de las experiencias propias de los otros. La experiencia actual aprovecha algo de las anteriores y contribuye para el perfeccionamiento de acciones posteriores, lo que defiende la idea de que el profesor debe 
construir su conocimiento por la reflexión sobre la práctica. (p. 76)

Surgen, entonces, en este panorama, nuevos retos para los profesores específicamente relacionados con tres aspectos: la planeación de las actividades para estudiantes en modalidades presenciales, virtuales ehíbridas; definición de roles al interior de las actividades de clase y las estrategias y herramientas de evaluación.

\section{Nuevos retos}

Así como las condiciones sociales y culturales cambian de manera constante, las instituciones de educación están llamadas a mutar. El profesor, responsable entre otras de la organización de la enseñanza (Pérez, 2020), estállamado a (re)pensar y movilizar nuevas estrategias de tipo didáctico y metodológico con el fin de atender a las necesidades particulares de sus contextos reales. Entre los principales aspectos que el profesor debería considerar de manera constante se encuentran:

(i) la planeación de sus actividades de enseñanza: el hecho de reconocer las condiciones particulares de las instituciones y de los estudiantes convoca a los profesores a delimitar de manera pertinente la planeación de las actividades. En este sentido el profesor puede responder a preguntas como son: ¿de qué medios dispone la institución?; ¿de qué medios dispone el estudiante?; ¿qué herramientas o metodologías de enseñanza favorecen la construcción de los saberes?; ¿cómo se vincula esta planeación con el modelo pedagógico que sustenta las actividades?; ¿qué competencias y saberes se desean desarrollar a través del curso?

(ii) Establecimiento de roles de profesores y estudiantes: si bien cada paradigma pedagógico sustenta unas compresiones sobre la forma en que los estudiantes aprenden, es claro que cada institución y cada profesor deben tener claro qué rol asumen en cada una de sus clases y cómo estos roles aportan al aprendizaje de sus estudiantes. Claramente, lo que se busca es quelos estudiantes no se sientan alienados frente al saber o instrumentalizados frente a los requerimientos de los profesores. El rol de los estudiantes, lejos de ser activo, debe mostrar a través de sus actividades de aprendizaje un interés por apropiarse de los saberes culturales necesarios para interactuar con sus pares en la sociedad.

(iii) Herramientas y mecanismos de evaluación: de la mano de los dos aspectos anteriores, aparece la evaluación como el seguimiento y valoración de los aprendizajes de los estudiantes. Si bien se han realizado estudios exhaustivos sobre las formas de evaluación, un diseño metodológico debería pasar por preguntas como: ¿a quién se va a evaluar?; ¿qué se va a evaluar?; ¿cuándo se va a evaluar?; ¿qué se espera en la evaluación?; ¿qué herramientas favorecen más el seguimiento a los aprendizajes en este curso?; ¿qué sujetos pueden participar en el contexto evaluativo, aparte del profesor?

Tener en cuenta estos aspectos, entre otros, aportaría a que tanto profesores como estudiantes comprendan que la construcción y apropiación de los saberes constituidos culturalmente responden a actividades planeadas en el marco de un proyecto pedagógico que también atiende a las nuevas necesidades de la sociedad.

\section{Referencias}

Alarcão, I. (1996). Ser professor reflexivo. En Formação reflexiva de professores, estratégias de supervisão (pp. 171-189). Oporto, Portugal: Porto Editora.

Almeida, M. e Pimenta, S. (2014). Pedagogia universitária - Valorizando o ensino e a docência na universidade. Revista Portuguesa de Educação, 27(2), 7-31.

Biggs, J. and Tang, C. (2011). Teaching for Quality Learning at University. What the Student Does. New York, USA: McGraw Hill.

Davídov, V. y Márkova, A. (1987). La concepción de la actividad de estudio de los escolares. En Shuare, M. (Org.), La psicología evolutiva y pedagógica en la URSS. Antología (pp. 316-344). Moscú, Rusia: Editorial Progreso.

Davídov, V. (1988). La enseñanza escolar y el desarrollo psíquico. Investigación psicológica teórica y experimental. Moscú, Rusia: Editorial Progreso. 
Grillo, M. (2000). O lugar da reflexão na construção do conhecimento profissional. En Morosini, M. (Org.), Professor de ensino superior. Identidade, docência e formação (pp. 75-80). Brasília, Brasil: Instituto Nacional de Estudios e Investigaciones Educativas "Anísio Teixeira".

Guzmán, G. y Quimbayo, C. (2012). Docencia universitaria. Reflexión pedagógica. Ibagué, Colombia: Universidad del Tolima.

Imbernón, F. (1994). La formación y el desarrollo profesional del profesorado. Hacia una nueva cultura profesional. Barcelona, España: Graó.

Junges, K. e Behrens, M. (2016). Uma formação pedagógica inovadora como caminho para a construção de saberes docentes no Ensino Superior. Educar em Revista, 59, 211-229.

Moretti, V. e Moura, M. (2010). O sentido em movimento na formação de professores de matemática. Zetetiké, 18(34), 155-180.

Morosini, M. (Org.) (2000). Docência universitária e os desafios da realidade nacional. En Professor do ensino superior. Identidade, docência e formação (pp. 11-20). Brasília, Brasil: Instituto Nacional de Estudios e Investigaciones Educativas "Anísio Teixeira".

Pérez, D. (2020). Organización de la enseñanza del profesor que enseña matemáticas en una escuela de administración: una posibilidad a partir de estudios de caso (tesis de posgrado). Doctorado en Educación, Facultad de Educación, Universidad de Antioquia.

Scott, C. (2015). El futuro del aprendizaje 2 ¿Qué tipo de aprendizaje se necesita en el siglo XXI? Investigación y prospectiva en educación. Recuperado de https:// unesdoc.unesco.org/ark:/48223/pf0000242996_spa.

Silva, T. (2010). Documentos de identidade. Uma introdução ás teorias do currículo. Belo Horizonte, Brasil: Autêntica.

Stößlein, A. and Kanet, J. (2016). Undergraduate research experiences: Identifying lessons learned and challenges for business schools. The International Journal of Management Education, 14(3), 349-367.

Zabalza, M. (2004). La enseñanza universitaria. El escenario y sus protagonistas. Madrid, España: Narcea, S.A. de Ediciones. 